\title{
Contact Formation Dynamics: Mapping Chemical Bond Formation between a Molecule and a Metallic Probe
}

\author{
Borislav Naydenov, Lucile C. Teague, ${ }^{\dagger}$ Peter Ryan, and John J. Boland ${ }^{\star}$ \\ School of Chemistry and the Centre for Research on Adaptive Nanostructures and \\ Nanodevices (CRANN), Trinity College, Dublin 2, Ireland
}

Received June 6, 2006; Revised Manuscript Received July 7, 2006

\begin{abstract}
We present a study that maps out chemical bond formation between a Pt-inked probe and a single 1,3-cyclohexadiene (1,3-CHD) molecule on $\mathrm{Si}(100)$. By separating the mechanical and electronic contributions to the current during the approach to contact, we show that there are significant forces between the probe and the $\mathrm{C}=\mathrm{C}$ of the molecule and we track the relaxation of the molecule, the emergence of a chemical bond feature in the LDOS, and the quenching of specific molecular vibrations during bond formation.
\end{abstract}

Understanding the detailed dynamics of chemical bond formation at surfaces is essential to a wide range of technologies. For example, the prospects for future molecular electronic devices ${ }^{1-13}$ hinge on the ability to form reproducible electrical contacts with single molecules..$^{2,3,14}$ Little is known about how individual molecules relax or how potentially important device properties such as the HOMOLUMO gap are modified by the contacting process. Similarly, the operation of catalysts and enzymes depends on understanding how particular molecules react with surface catalytic sites. ${ }^{15-17}$ The manner in which molecules engage with and ultimately dock at these surface sites is critically important. In these and related systems, numerous studies have reported on the final state properties, that is, after the contact has been assembled or the reaction completed. The actual dynamics of chemical bond formation is poorly understood and represents a serious impediment to the rational design of the materials and devices that underpin these technologies.

Here we present a study that maps out chemical bond formation as a Pt-inked probe approaches and makes contact with a single 1,3-cyclohexadiene (1,3-CHD) molecule on Si(100). By explicitly separating the mechanical and electronic contributions to the current during the approach, we show that there are significant forces ${ }^{18}$ between the probe and $\mathrm{C}=$ $\mathrm{C}$ region of the molecule and we track both the relaxation and rehybridization of the molecule, the emergence of a chemical bonding feature in the LDOS, and the quenching of specific molecular vibrations, all in excellent agreement with density functional theory (DFT) calculations. We

\footnotetext{
* Corresponding author. E-mail: jboland@tcd.ie.

† Present address: Surface and Microanalysis Sciences Division, National Institute of Standards and Technology, 100 Bureau Drive Mailstop 8372, Gaithersburg, MD 20899
}

envision that these methods will be useful for identifying candidate metal-molecule systems for device applications and for addressing the operation and selectivity of certain catalysts.

Contact and bond formation was studied using a cryogenic STM (Createc) in which a single-crystal $\operatorname{Pt}(111)$ metal sample and a $\mathrm{Si}(100)$ substrate $[n+$-type As, $<5 \mathrm{~m} \Omega \cdot \mathrm{cm}$ ] were placed in a sample holder that allows each to be heated and prepared separately. Following characterization by LEED, both samples were simultaneously exposed at room temperature to less than $0.1 \%$ of a monolayer of 1,3-CHD, transferred into the STM, and cooled to $5 \mathrm{~K}$. Tungsten STM probes were cleaned by electron bombardment and then "inked" with atoms from the Pt substrate using a method described previously ${ }^{18}$ and which provided control over the composition of the probe. The Pt-inked probe was then carefully brought into tunneling contact with the $\mathrm{Si}(100)$ surface, and topographic images and spectroscopic measurements were recorded.

The close-up STM image in Figure 1a reveals buckled dimers in weakly ordered $\mathrm{c}(4 \times 2)$ and $\mathrm{p}(2 \times 2)$ domains (dashed boxes in Figure 1a) together with a single 1,3-CHD molecule (bright feature) bound to the surface in the $[2+$ 2] intradimer configuration. ${ }^{19}$ The STM probe tip was positioned over the molecule's maximum (black cross in Figure 1a) known to be associated with the single remaining $\mathrm{C}=\mathrm{C}$ bond in the adsorbed molecule,$^{20}$ and the local density of states $(\mathrm{LDOS})^{21}$ at different probe-molecule separations was recorded using a $15 \mathrm{mV}$ modulation at $534 \mathrm{~Hz}$ during the bias sweep. Figure $1 \mathrm{~b}$ is a map of the empty LDOS in the energy range from 100 to $700 \mathrm{meV}$ recorded as a function of the probe height above the molecule. The filled LDOS in 


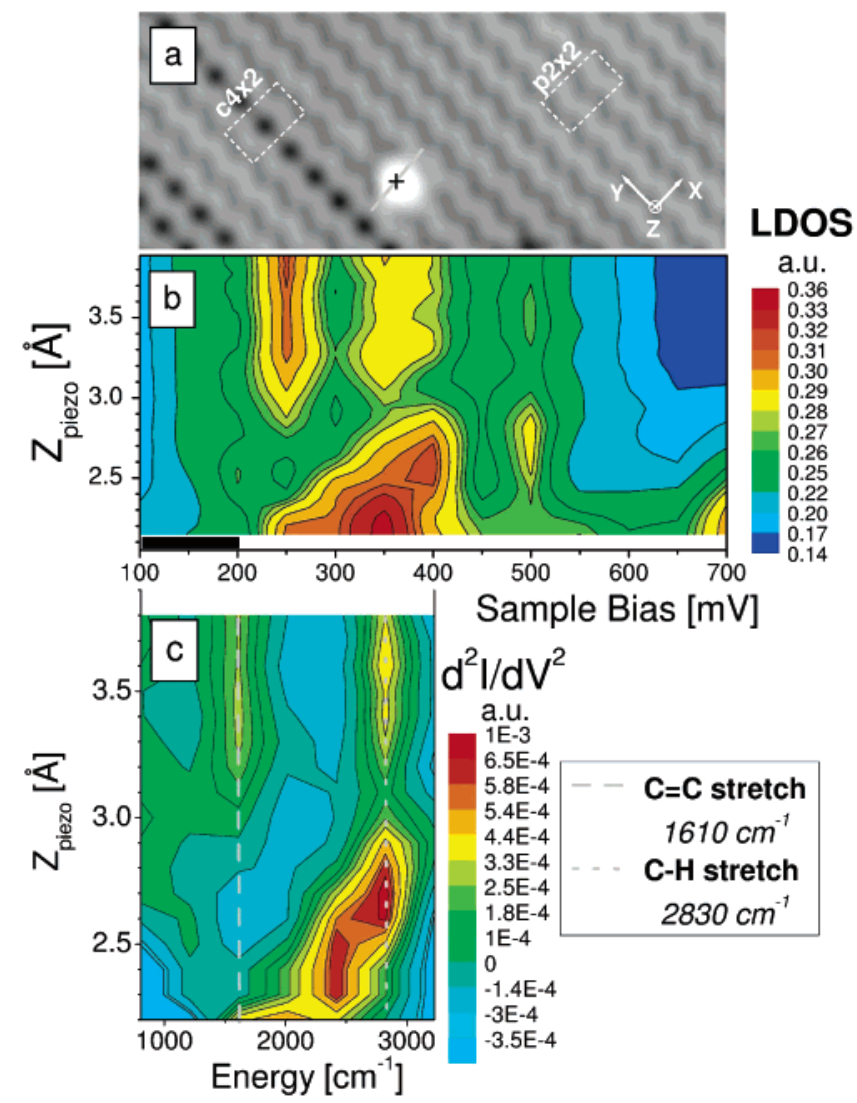

Figure 1. Density of states map of the probe-molecule contact formation. (a) Topographic image of the 1,3-cyclohexadiene molecule adsorbed on the $\mathrm{Si}(100)$ surface recorded with $0.1 \mathrm{pA}$ tunneling current and sample bias of $700 \mathrm{mV}$ at $5 \mathrm{~K}$. Area size $130 \times 100 \AA$. The dashed boxes comprise domains with $\mathrm{c} 4 \times 2$ and $\mathrm{p} 2 \times 2$ Si-surface reconstructions. The position where the LDOS spectra were taken is marked with a black cross. The gray solid line indicates the cross section across the molecule along which the relaxation spectroscopy in Figure 2 was recorded. (b) The electron local density of states $(\operatorname{LDOS}=(\mathrm{d} I / \mathrm{d} V) /(I / V))$ dependence on the probe-molecule separation is presented as a color map. The black horizontal bar on the bottom left shows a region of LDOS that is essentially independent of separation. The emergence of the electronic state at $350 \mathrm{mV}$ associated with bond formation is clearly visible. (c) Inelastic electron tunneling spectroscopy (IETS, $\mathrm{d}^{2} I /$ $\mathrm{d} V^{2}$ ) at different probe-molecule separations shows the presence of two molecule vibrational bands that are quenched during the approach.

this energy range is not shown but is essentially featureless, reflecting the combination of a wide HOMO-LUMO gap, the Si band gap, and degenerately n-doped substrate (see Supporting Information no. 1). (The absolute separation in Figure $1 b$ is unknown, and the zero was chosen with the aid of computation, see below). In the energy range up to 200 $\mathrm{meV}$, the LDOS is essentially independent of probemolecule separation. However, at large separations there is a weak doublet feature centered about $280 \mathrm{meV}$ that is due to inelastic losses associated with the molecule on the surface (see below). Upon closer approach these features are quenched and an intense broad peak emerges in the LDOS at $350 \mathrm{meV}$ (Figure 1b). Within this range of probemolecule separations, the emergence and disappearance of this $350 \mathrm{meV}$ peak is completely reversible and the amplitude of the peak falls off sharply with distance from the $\mathrm{C}=\mathrm{C}$ topographical maximum (see Supporting Information no. 2). Moreover, measurements recorded at separations below that in Figure $1 \mathrm{~b}$ or at biases above $\pm 0.8 \mathrm{~V}$ tended to perturb the molecule and surrounding dimers, resulting in a measurable hysteresis. ${ }^{18}$

Figure 1c shows the second derivative spectrum $\left(\mathrm{d}^{2} I / \mathrm{d} V^{2}\right)$ as a function of probe-molecule separation. At large separation, there are two clearly defined vibrational features ${ }^{22}$ at 1610 and $2830 \mathrm{~cm}^{-1}$ assigned to the stretching modes of the $\mathrm{C}=\mathrm{C}$ double bond and $\mathrm{C}-\mathrm{H}$ bonds, respectively. These features are quenched upon closer approach and accompanied by the emergence of the $350 \mathrm{meV}$ LDOS feature. This anticorrelation between the LDOS and vibration peaks is reversible, with the former being recovered at large separations. On the basis of these data, we conclude that the probe forms a chemical bond with the $\mathrm{C}=\mathrm{C}$ of the molecule at small separations, quenching the $\mathrm{C}=\mathrm{C}$ vibration and generating a new electronic feature associated with this bond.

To demonstrate that bond formation occurs at the $\mathrm{C}=\mathrm{C}$ region of the molecule, current-distance $(I-Z)$ spectroscopic studies ${ }^{18,23}$ were performed to directly measure the interaction force, $F$, between the probe and the $\mathrm{C}=\mathrm{C}$. Using a $0.01 \mathrm{~nm}$ dither at $534 \mathrm{~Hz}$ applied to the $Z$ piezo under open-loop conditions, the current and the first $(\mathrm{FH})$ and second harmonics $(\mathrm{SH})\left(\mathrm{d} I / \mathrm{d} Z\right.$ and $\left.\mathrm{d}^{2} I / \mathrm{d} Z^{2}\right)$ were measured simultaneously using a lock-in as the probe was approached to and retracted from the surface. To eliminate LDOS contributions at different probe-molecule separations, we recorded all measurements at $200 \mathrm{meV}$ sample bias because the LDOS within the tunneling energy window defined by this bias is constant and independent of separation (see Figure 1b). This interaction force, $F$, induces a vertical displacement in the $\mathrm{C}=\mathrm{C}\left(\Delta Z_{\mathrm{C}=\mathrm{C}}\right)$ that is detectable in the current. The simplest approach is to assume that the force is zero at large separations, so the current is described by

$$
I_{F=0} \approx \exp \left(-A \phi^{1 / 2} Z\right) \quad \text { large } Z ; F=0 ; Z=Z_{\mathrm{p}}
$$

and

$$
I_{F \neq 0} \approx \exp \left[-A \phi^{1 / 2}\left(Z_{\mathrm{p}}+k_{\mathrm{S}}{ }^{-1} F\right)\right] ; \quad F=-k_{\mathrm{S}} \Delta Z_{\mathrm{C}=\mathrm{C}}
$$

where $\phi$ is the tunneling barrier height, $A=2 \pi\left(8 m_{\mathrm{e}}\right)^{1 / 2} / \mathrm{h}$, $Z_{\mathrm{p}}=Z+\Delta Z_{\mathrm{C}=\mathrm{C}}$ is the piezo-driven probe-surface separation, and $k_{\mathrm{S}}$ is the spring constant associated with the attachment of the $\mathrm{C}=\mathrm{C}$ to the surface. Combining eqs 1 and 2, we obtained the force spectrum

$$
F\left(Z_{\mathrm{p}}\right)=\left[\ln \left(I_{F=0}\right)-\ln \left(I_{F \neq 0}\right)\right]\left(A \phi^{1 / 2} k_{\mathrm{S}}^{-1}\right)^{-1},
$$

where $I_{F=0}\left(Z_{\mathrm{p}}\right)$ was obtained by fitting the experimental spectrum at large separations and extrapolating it to smaller values, whereas for $I_{F \neq 0}\left(Z_{\mathrm{p}}\right)$ the actual experimental data were used. Figure 2 shows a map of $F\left(Z_{\mathrm{p}}\right)$ as the probe approaches the surface, recorded from different lateral positions along the cross section in Figure 1a. Figure 2 shows that the force spectrum has near topographic spatial resolution and is 


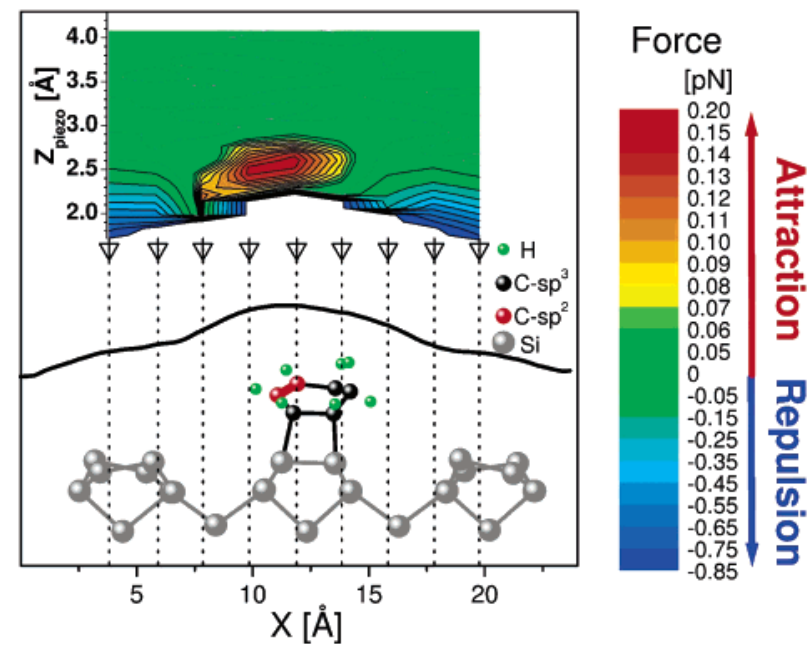

Figure 2. Spatial map of the tip-sample interaction force. The insert on the top shows a $F\left(Z_{\mathrm{p}}\right)$ map along the gray solid line in Figure 1a obtained by applying eq 3 with $200 \mathrm{mV}$ sample bias and parameters obtained in ref 18 . The map was constructed by averaging spectra recorded at individual positions (arrows) along the dotted lines and assuming fixed $k_{\mathrm{s}}=100 \mathrm{~N} / \mathrm{m} .{ }^{18}$ The solid black line presents the surface corrugation $(0.1 \mathrm{pA}, 700 \mathrm{mV})$ along the cross section taken as in Figure 1a. The topographic maximum is exactly above the $\mathrm{C}=\mathrm{C}$ double bond as evidenced from the model of the molecule and the surface shown on the bottom of the figure. This maximum coincides with the well-defined attraction feature (red) in the $F\left(Z_{\mathrm{p}}\right)$ map and is the position where the LDOS and IETS maps (see Figure $1 b$ and c) were recorded.

sensitive to the presence of the adsorbed 1,3-CHD molecule. In particular, there is significant attraction (red feature in
Figure 2) at the location of the molecules' $\mathrm{C}=\mathrm{C}$ bond, consistent with a local chemical bonding interaction.

A more detailed force analysis is possible using the $\mathrm{FH}$ and SH signals, which are sensitive to subtle variations in the current, and for which it was previously shown that changes in the $\mathrm{FH}$ and $\mathrm{SH}$ signals during the approach reflect local changes in the force gradients that act between the probe and the corresponding surface location. ${ }^{18}$ By analyzing the current signal and its harmonics with the probe initially positioned above the $\mathrm{C}=\mathrm{C}$ bond, the subsequent relaxation of the molecule during the approach to bond formation was measured $^{18}$ and plotted in Figure $3 \mathrm{a}$ (dashed line). At large separations, the $\mathrm{C}=\mathrm{C}$ moiety is weakly attracted toward the probe. When the probe advances further, the $\mathrm{C}=\mathrm{C}$ pulls away, but then as the $350 \mathrm{meV}$ state emerges (see inset Figure $3 \mathrm{a}$ ), the $\mathrm{C}=\mathrm{C}$ moiety is firmly drawn toward the probe. Beyond this point, the continued motion of the probe toward the surface is tracked by the $\mathrm{C}=\mathrm{C}$ moiety, which is ultimately compressed (blue dashed line section, Figure 3a) beyond its neutral position $\left(\Delta Z_{C=C}=0\right)$. No other region of the molecule exhibited this behavior, demonstrating that the spectroscopic map in Figure 2 is sensitive to details of the probe-molecule interaction.

These data clearly show that the emergence of the 350 $\mathrm{meV}$ state during the approach to contact is associated with a specific bonding interaction between the probe and the $\mathrm{C}=$ $\mathrm{C}$ bond of the molecule. This was confirmed by DFT calculations that involved a 1,3-CHD molecule attached to a $\mathrm{Si}(100)$ dimer with a $\mathrm{Pt}$ probe (modeled as a five atom (100) cluster constrained to the bulk structure) positioned

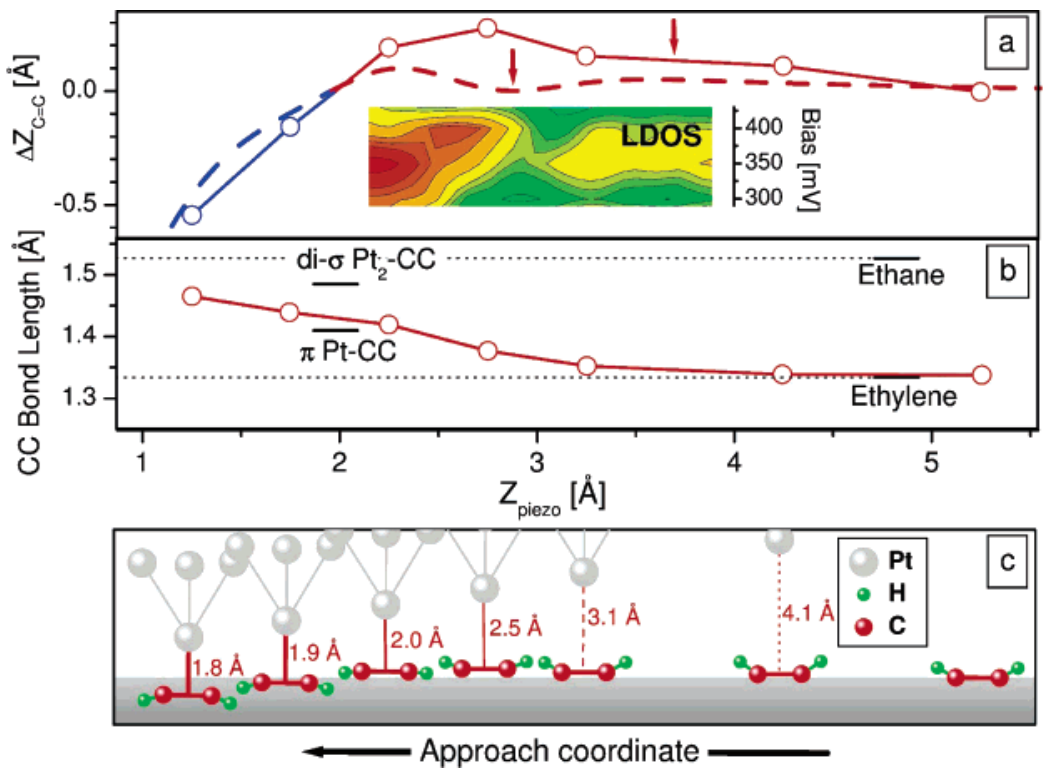

Figure 3. $\mathrm{C}=\mathrm{C}$ double-bond relaxation and rehybridization during the probe-molecule contact formation. (a) The DFT calculated displacement $\left(\Delta Z_{C=C}\right)$ of the $C=C$ bond relative to the equilibrium position (zero net force) is shown with an open circle and solid line. The attraction and repulsion between the probe and the $\mathrm{C}=\mathrm{C}$ bond are presented as red and blue, respectively. The experimentally derived $\Delta Z_{\mathrm{C}=\mathrm{C}}$ is plotted as a dashed line and is a good match to the theoretical curve. The inset is an aligned strip of the LDOS map showing the appearance of the new electronic state after the local minimum (arrow on dashed curve). (b) The DFT calculated C $=\mathrm{C}$ bond length (open circle and solid line) during the probe approach is compared with calculations for the ethylene adsorption on a Pt surface. ${ }^{27} \mathrm{The}$ comparison indicates that the probe-molecule bond can be assigned as a $\pi \mathrm{Pt}-\mathrm{CC}$ bond. (c) The calculated atom positions along the approach coordinate showing the rehybridization of the carbon atoms and concomitant displacement of the hydrogen atoms. The gray boundary corresponds to the position of zero force $\left(\Delta Z_{\mathrm{C}=\mathrm{C}}=0\right)$. 
over the $\mathrm{C}=\mathrm{C}$. The $\mathrm{Si}(100)$ surface was modeled using a three-dimer-long $3 \times 2$ slab, six-layers deep and terminated by $\mathrm{H}$ atoms on the lower surface. Plane wave (cutoff energy $240 \mathrm{eV}$ ) and the PW-91 GGA methods ${ }^{24}$ were used with 1 $\times 3 \times 2$ Monkhorst-Pack grid ${ }^{25}$, for which the energy and maximum force convergences were set to $2 \times 10^{-5} \mathrm{eV} /$ atom and $0.05 \mathrm{eV} / \AA$, respectively. ${ }^{26}$ With the probe located $2 \mathrm{~nm}$ away and the bottom two Si layers constrained to the bulk, the molecule-surface system was relaxed to determine the total energy of the isolated systems. All Si atoms were then fixed at their optimized positions, and the molecule was allowed to relax as the probe approached the $\mathrm{C}=\mathrm{C}$ bond.

On the basis of these DFT results, the change in the $Z$ position and bond length of the $\mathrm{C}=\mathrm{C}$ during the approach were calculated and shown in Figure $3 \mathrm{a}$ and $\mathrm{b}$ (solid lines connecting open circles) while Figure $3 \mathrm{c}$ schematically describes the relaxation of the $\mathrm{C}=\mathrm{C}$ moiety and its constituent atoms. Initially, the trigonal plane of the $\mathrm{H}-\mathrm{C}=\mathrm{C}-\mathrm{H}$ moiety is tilted up from the surface plane and the $\mathrm{C}=\mathrm{C}$ bond length is similar to that in ethylene. As the probe approaches, the entire moiety is attracted toward it and the length of the $\mathrm{C}=\mathrm{C}$ bond begins to increase from 1.337 to $1.345 \AA$. This is followed by a pause during which the $\mathrm{C}=\mathrm{C}$ does not respond to the approaching probe but after which there is attraction that results in further bond elongation and local rehybridization as the $=\mathrm{C}-\mathrm{H}$ bonds flip down toward the surface (see Figure $3 \mathrm{a}$ and $\mathrm{c}$ ). The formation of a stable chemically bonded contact between the probe and the $\mathrm{C}=\mathrm{C}$ occurs when there is no net force and for which the $\mathrm{C}=\mathrm{C}$ returns to its original position above the surface $\left(\Delta Z_{C}=C=\right.$ 0 in Figure $3 b$ and the gray boundary in Figure 3c). At this equilibrium position, the length of the $\mathrm{C}=\mathrm{C}$ closely matches that found in $\mathrm{p} \pi \rightarrow \mathrm{d} \pi$ complexes between ethylene and single Pt surface atoms. ${ }^{27}$ As the probe approaches further, the contact bond is compressed and there is a commensurate elongation of the $\mathrm{C}=\mathrm{C}$ bond.

The relaxation of the $\mathrm{C}=\mathrm{C}$ moiety predicted by computation closely matches that seen in the experiment (solid vs dashed curve in Figure 3a). The $Z$ scale in this and all figures has been set by aligning the zero force points (the energy minima) of the calculated and experimental approach curves. One notable difference is that the experimental curve shows that the $\mathrm{C}=\mathrm{C}$ bond relaxes back toward its neutral position prior to bond formation (accompanied by quenching of the $\mathrm{C}=\mathrm{C}$ vibration and the emergence of the $350 \mathrm{meV}$ peak in the LDOS), whereas theory shows only a reduced attraction with the probe (see arrows in Figure 3a) but at larger separations. Although this difference may in part reflect our inability to perform fully relaxed calculations, an analysis of the molecule's coordinates clearly shows that this behavior is associated with the rehybridization of the $\mathrm{C}=\mathrm{C}$ bond (Figure 3c). In the experimental model, this relaxation is attributed to either a small barrier to bond formation ${ }^{18}$ or a local increase in the spring constant that tethers the molecule to the surface. It is not possible at present to distinguish between these possibilities, but both are consistent with a reorganization of the molecule prior to actual bond formation.

On the basis of these data, the emergence of the $350 \mathrm{meV}$ chemical bonding signature in the LDOS in Figure $1 \mathrm{~b}$ is associated with the rehydridization of the $\mathrm{C}=\mathrm{C}$ bond. The unoccupied $350 \mathrm{meV}$ peak should then correspond to the antibonding level associated with the $\mathrm{p} \pi \rightarrow \mathrm{d} \pi$ bonding interaction, in which $\pi$ electron density of the $\mathrm{C}=\mathrm{C}$ is transferred to an empty $\mathrm{d}$ orbital on the Pt probe. ${ }^{27}$ This assignment is consistent with quenching of the $\mathrm{C}=\mathrm{C}$ vibration (Figure 1c) and is reminiscent of the $\mathrm{p} \pi \rightarrow \mathrm{d} \pi$ complex observed in the case of ethylene on $\operatorname{Pt}(111)$ and for which NEXAFS studies show an empty $\pi^{*}$ level located about 300 meV above $E_{\mathrm{f}}{ }^{28}$ On the $\mathrm{Pt}(111)$ surface this complex is known to be unstable above $50 \mathrm{~K}$ and to convert to di-sigma bonded structure involving two Pt atoms. ${ }^{29}$ However, it is likely that the latter structure is precluded here because it requires two $\mathrm{Pt}$ atoms almost equidistant from the $\mathrm{C}=\mathrm{C}$, which would be immediately evident in the present case because of the grossly distorted STM images that would result.

The demonstrated ability to track the relaxation of a single molecule and the evolution of its electronic and vibrational properties over a range of separations, up to and including the point of chemical bond formation, has important implications. This method has important advantages over the conventional AFM technique, which does not have these same spectroscopic capabilities. It results from an ability to explicitly separate the mechanical and electronic contributions to the current, which will always be possible for molecules with large HOMO-LUMO gaps and semiconductors where the location of the Fermi energy can be controlled by doping. ${ }^{13}$ When combined with the subpicometer control of cryogenic STM, this approach provides snapshots of the dynamics along the reaction coordinate. For molecular electronics, this facilitates a systematic approach to evaluate, in combination with different contacting metals, the performance of candidate molecules in different environments and bonding configurations, and represent an important first step toward the rational design of molecular devices and their operation. In the case of catalysis, it provides a methodology to examine the dynamics of chemical bond formation at different active sites, and in combination with molecular manipulation and attachment using STM probes,${ }^{30}$ the ability to measure the local affinity of a substrate toward different molecules.

Acknowledgment. This work was supported by Science Foundation Ireland under grant 00/PI.1/C077A.

Supporting Information Available: Supporting Information no. 1: LDOS spectra of the 1,3-CHD molecule and the bare region of the $\mathrm{Si}(100)$ surface recorded at a large probemolecule separation. Supporting Information no. 2: lateral confinement of the LDOS state associated with bond formation. This material is available free of charge via the Internet at http://pubs.acs.org.

\section{References}

(1) Muller, C. J.; Vleeming, B. J.; Reed, M. A.; Lamba, J. J. S.; Hara, R.; Jones, L.; Tour, J. M. Nanotechnology 1996, 7, 409-411.

(2) Cui, X. D.; Primak, A.; Zarate, X.; Tomfohr, J.; Sankey, O. F.; Moore, A. L.; Moore, A. T.; Gust, D.; Harris, G.; Lindsay, S. M. Science 2001, 294, 571-574. 
(3) Nitzan, A.; Ratner, M. A. Science 2003, 300, 1384-1389.

(4) Xu, B.; Tao, N. Science 2003, 301, 1221-1223.

(5) Haiss, W.; Van Zalinge, H.; Higgins, S. J.; Bethell, D.; Höbenreich, H.; Schiffrin, D. J.; Nichols, R. J. J. Am. Chem. Soc. 2003, 125 , $15294-15295$

(6) Reed, M. A.; Zhou, C.; Muller, C. J.; Burgin, T. P.; Tour, J. M. Science 1997, 278, 252-254.

(7) Blum, A. S.; Kushmerick, J. K.; Long, D. P.; Patterson, C. H.; Yang, J. C.; Henderson, J. C.; Yao, Y. X.; Tour, J. M.; Shashidhar, R.; Ratna, B. R. Nat. Mater. 2005, 4, 167-172.

(8) Gimzewski, J. K.; Joachim, C. Science 1999, 283, 1683-1688

(9) Gittins, G. I.; Bethell, D.; Schiffrin, D. J.; Nichols, R. J. Nature 2000 408, 67-69.

(10) Maruccio, G.; Cingolani, R.; Rinaldi, R. J. Mater. Chem. 2004, 14, $542-554$.

(11) Guisinger, N. P.; Yoder, N. L.; Hersam, M. C. PNAS 2005, 102, $8838-8843$.

(12) Xue, Y. Q.; Ratner, M. A. Int. J. Quantum Chem. 2005, 102, 911924.

(13) Rakshit, T.; Liang, G.-C.; Ghosh, A. W.; Datta, S. Nano Lett. 2004, 4, 1803-1807.

(14) Hipps, K. W. Science 2001, 294, 536-537.

(15) Topsøe, H.; Hinnemann, B.; Nørskov, J. K.; Lauritsen, J. V.; Besenbacher, F.; Hansen, P. L.; Hytoft, G.; Egeberg, R. G.; Knudsen, K. G. Catal. Today 2005, 107-08, 12-22.

(16) Lauritsen, J. V.; Vang, R. T.; Besenbacher, F. Catal. Today 2005, $111,34-43$.

(17) Weigelt, S.; Busse, C.; Petersen, L.; Rauls, E.; Hammer, B.; Gothelf, K. V.; Besenbacher, F.; Linderoth, T. R. Nat. Mater. 2006, 5, 112117
(18) Naydenov, B.; Ryan, P.; Teague, L. C.; Boland, J. J., to be submitted for publication (submitted to Phys. Rev. Lett and provided as RelatedWork.pdf)

(19) Teague, L. C.; Boland, J. J. J. Phys. Chem. B 2003, 107, 38203823 .

(20) Teague, L. C.; Boland, J. J. Thin Solid Films 2004, 464-465, 1-4

(21) Scanning Tunneling Microscopy and Spectroscopy: Theory, Techniques, and Applications; Bonnell, D. A., Ed.; VCH: New York, 1993.

(22) Stipe, B. C.; Rezaei, M. A.; Ho, W. Science 1998, 280, 1732-1736.

(23) Chen, C. J.; Hamers, R. J. J. Vac. Sci. Technol., B 1991, 9, 503505.

(24) Perdew, J. P.; Zunger, A. Phys. Rev. B 1981, 23, 5048-5079.

(25) MacDonald, A. H. Phys. Rev. B 1978, 18, 5897-5899.

(26) Segall, M. D.; Lindan, P. J. D.; Probert, M. J.; Pickard, C. J.; Hasnip, P. J.; Clark, S. J.; Payne, M. C. J. Phys.: Condens. Matter 2002, 14, 2717-2744.

(27) Watson, G. W.; Wells, R. P. K.; Willock, D. J.; Hutchings, J. J. Phys. Chem. B 2000, 104, 6439-6446.

(28) Cassuto, A.; Mane, M.; Jupille, J. J. Phys. Chem. 1992, 96, 59875993. The $\pi^{*}$ energy value above the Fermi level used in the manuscript is estimated by subtracting the $\mathrm{C} 1 \mathrm{~s}$ binding energy from the energy of the $1 \mathrm{~s}-\pi^{*}$ resonance; the later values are taken from ref 28. This estimate is valid because of the self-screened final state of the $1 \mathrm{~s}-\pi^{*}$ resonance. ${ }^{28}$

(29) Cassuto, A.; Mane, M.; Jupille, J. Surf. Sci. 1991, 249, 8-14.

(30) Dudko, O.; Filippov, A. E.; Klafter, J.; Urbakh, M. Nano Lett. 2003 3, 795-798.

NL061294A 